Marc Feuilloley, Pierre Netchitaïlo, Catherine Delarue, François Leboulanger, Georges Pelletier, Hubert Vaudry

\section{Quels sont les rôles du cytosquelette dans les processus de sécrétion des hormones stéroïdes?}

La libération des hormones stéroïdes ne semble pas nécessiter une étape d'empaquetage comme dans le cas des hormones peptidiques. C'est pourquoi le rôle du cytosquelette dans les cellules stéroïdogènes a été longtemps ignoré. Les résultats accumulés depuis ces dernières années permettent cependant d'établir un schéma général concernant les rôles respectifs des différents éléments du cytosquelette dans la biosynthèse et la libération des hormones stéroïdes. Ainsi peut-on dire que les microtubules et les filaments intermédiaires semblent être impliqués dans la transduction des messages au niveau de la membrane cytoplasmique alors que les microfilaments interviendraient dans le mécanisme de biosynthèse des stéroïdes.

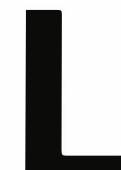

e cytosquelette est composé d'au moins trois familles de fibres, les microtubules, les microfilaments et les filaments intermédiaires $\left(m / s n^{\circ} 1\right.$, vol. $5, p .24$ 32) dont le diamètre et la composition sont différents. C'est principalement en agissant sur le degré de polymérisation de ces fibres au moyen de drogues telles que la colchicine ou la vinblastine pour les microtubules, les cytochalasines pour les microfilaments ou le $\beta$ $\beta$ 'iminodipropionitrile (IDPN) pour les filaments intermédiaires que le rôle physiologique du cytosquelette a été étudié (Tableau I).

Il est maintenant bien établi que, dans les cellules endocrines peptidergiques, les microtubules et les microfilaments contrôlent le transport et l'exocytose des granules de sécrétion. Toutefois, les hormones stéroïdes ne semblent pas être l'objet d'une compartimentation et on considère généralement que ces molécules diffusent passivement au travers de la membrane cytoplasmique. C'est pourquoi le rôle du cytosquelette dans la production des hormones stéroïdes a été principalement étudié par des approches biochimiques.

\section{Microtubules et stéroïdogenèse}

La plupart des travaux réalisés chez les vertébrés montrent que les agents antimicrotubulaires n'affectent pas la production spontanée des hormones stéroïdes par les cellules corticosurrénaliennes, les cellules lutéales ou les cellules de la granulosa. En revanche, la colchicine stimule légèrement la production de testostérone par les cellules de Leydig, mais cette augmentation semble étroitement dépendante des conditions expérimentales [1]. Des études similaires effectuées sur la lignée de cellules corticosurrénaliennes tumorales Yl ont montré que la colchicine et la vinblastine stimulent la stéroïdogenèse basale. Cependant, les cellules Yl présentent la particularité, comme d'autres lignées de cellules stéroïdogènes tumorales, de ne posséder qu'une faible quantité de microtubules libres, la tubuline étant 
principalement complexée avec du cholestérol sous forme de granules cytoplasmiques [2]. De ce fait, en agissant sur la tubuline, les agents antimicrotubulaires libéreraient le cholestérol induisant ainsi une augmentation de la stéroïdogenèse basale.

La présence de microtubules ancrés dans la membrane cytoplasmique suggère que ces fibres pourraient être impliquées dans les mécanismes de couplage des signaux hormonaux à la réponse stéroïdogène. Ainsi, les agents antimicrotubulaires bloquent l'effet stimulateur de l'hormone lutéinisante ( $\mathrm{LH}$ ) sur les cellules de Leydig et la réponse des cellules de la granulosa à l'hormone folliculostimulante (FSH). La colchicine et la vinblastine inhibent aussi la réponse des cellules corticosurrénaliennes à l'ACTH et à la sérotonine, mais n'affectent pas l'activité corticotrope de l'AMPc ni celle de la forskoline et des ions fluorures, deux agents qui stimulent le système adénylcyclasique au niveau des sous-unités catalytiques et régulatrices, respectivement [3]. En revanche, la vinblastine ne modifie pas l'activité corticotrope de l'acétylcholine et de l'angiotensine II (dont les récepteurs sont couplés positivement à la phospholipase C et/ou négativement à l'adénylate cyclase). Ces résultats suggèrent donc que les microtubules sont impliqués dans l'interaction des récepteurs avec la sous-unité régulatrice stimulatrice de l'adénylate cyclase (Gs), mais n'interviennent pas dans les systèmes de transduction passant par une activation du turnover des phosphoinositides ou par une inhibition de l'activité adényl cyclasique via une protéine $\mathrm{Gi}(\mathrm{m} / \mathrm{s}$ suppl. au $n^{\circ} 10$, vol. 4, p. 40-48).

\section{Microfilaments et stéroïdogenèse}

Les cytochalasines n'affectent pratiquement pas la libération spontanée des stéroïdes par les cellules de la granulosa, les cellules lutéales et les cellules de Leydig. On peut donc penser que les microfilaments jouent un rôle mineur dans la production spontanée des stéroïdes sexuels. A l'inverse, dans les cellules corticosurrénaliennes, l'effet de la cytochalasine B et l'organisation des microfi$\mathrm{m} / \mathrm{s} n^{\circ} 9$ vol. 5 , novembre 89

\begin{tabular}{|c|c|c|}
\hline \multicolumn{2}{|c|}{ Tableau I } & $\begin{array}{l}\text { ABLES D'AFFECTER } \\
\text { J CYTOSQUELETTE }\end{array}$ \\
\hline & Agents dépolymérisants & Agents stabilisateurs \\
\hline Microtubules & $\left.\begin{array}{l}\text { Colchicine } \\
\text { Nocodazole } \\
\text { Colcémide } \\
\text { Vinblastine } \\
\text { Vincristine } \\
\text { Vinédipine } \\
\text { Maytansine }\end{array}\right\} \begin{array}{l}1 \text { site de liaison } \\
\text { /dimère de tubuline } \\
\text { /dimère de tubuline }\end{array}$ & $\begin{array}{c}\text { Isaxonine } \\
\text { (action peu spécifique) } \\
\text { Taxol }\end{array}$ \\
\hline Microfilaments & $\begin{array}{l}\text { Cytochalasines } \\
\text { (principalement B, D et E) }\end{array}$ & $\begin{array}{c}\text { Phalloïdine } \\
\text { (effet irréversible) }\end{array}$ \\
\hline $\begin{array}{l}\text { Filaments } \\
\text { intermédiaires }\end{array}$ & $\left.\begin{array}{l}\text { Orthovanadate de sodium } \\
\begin{array}{l}\text { Toxine diphtérique } \\
\text { Exotoxine A }\end{array} \\
\beta-\beta^{\prime} \text { iminodipropionitrile (IDPN) }\end{array}\right\} \begin{array}{l}\text { action } \\
\text { peu } \\
\text { ppécifique }\end{array}$ & \\
\hline
\end{tabular}

laments sont très variables selon les espèces et il est permis de penser que ces différences structurales et fonctionnelles seraient étroitement liées. Dans les cellules stéroïdogènes tumorales, l'effet de la cytochalasine $B$ dépend de la présence ou de l'absence de sérum dans le milieu de culture. En présence de sérum, la cytochalasine $B$ stimule la stéroïdogenèse basale et potentialise la réponse à l'ACTH. Au contraire, la cytochalasine B exerce un effet inhibiteur en l'absence de sérum. L'effet stimulateur de la cytochalasine B peut être mimé par l'addition de HDL (high density lipoprotein) au milieu de culture contenant la drogue, ce qui suggère que les microfilaments pourraient être impliqués dans l'incorporation de précurseurs de biosynthèse de: hormones stéroïdes présents dans le milieu de culture [4]. Il est maintenant bien établi que le réseau microfilamentaire est en étroite relation avec les récepteurs membranaires. Toutefois, le rôle principal des microfilaments dans les cellules stéroïdogènes semble se situer plus en aval dans le méca- nisme d'action des facteurs de stimulation. Dans les cellules de la granulosa, les cytochalasines inhibent non seulement l'effet stimulateur de la LH, mais aussi celui de son second messager. De même, dans les cellules de Leydig et dans les cellules corticosurrénaliennes, la cytochalasine $\mathrm{B}$ et les anticorps anti-actine bloquent la biosynthèse des stéroïdes en inhibant le transfert du cholestérol à la mitochondrie [5]. Toutefois, les microfilaments semblent aussi intervenir au niveau de l'hydroxylation de la 11-désoxycorticostérone (DOC) en corticostérone [6]. Il est donc permis de penser que dans les cellules corticosurrénaliennes les microfilaments sont impliqués non seulement dans le transport dı cholestérol, mais aussi dans le mécanisme de translocation de la DOC du réticulum endoplasmique à la mitochondrie.

\section{Filaments intermédiaires et stéroïdogenèse}

Le rôle des filaments intermédiaires dans les mécanismes de sécrétion est encore mal connu. Dans la plupart 


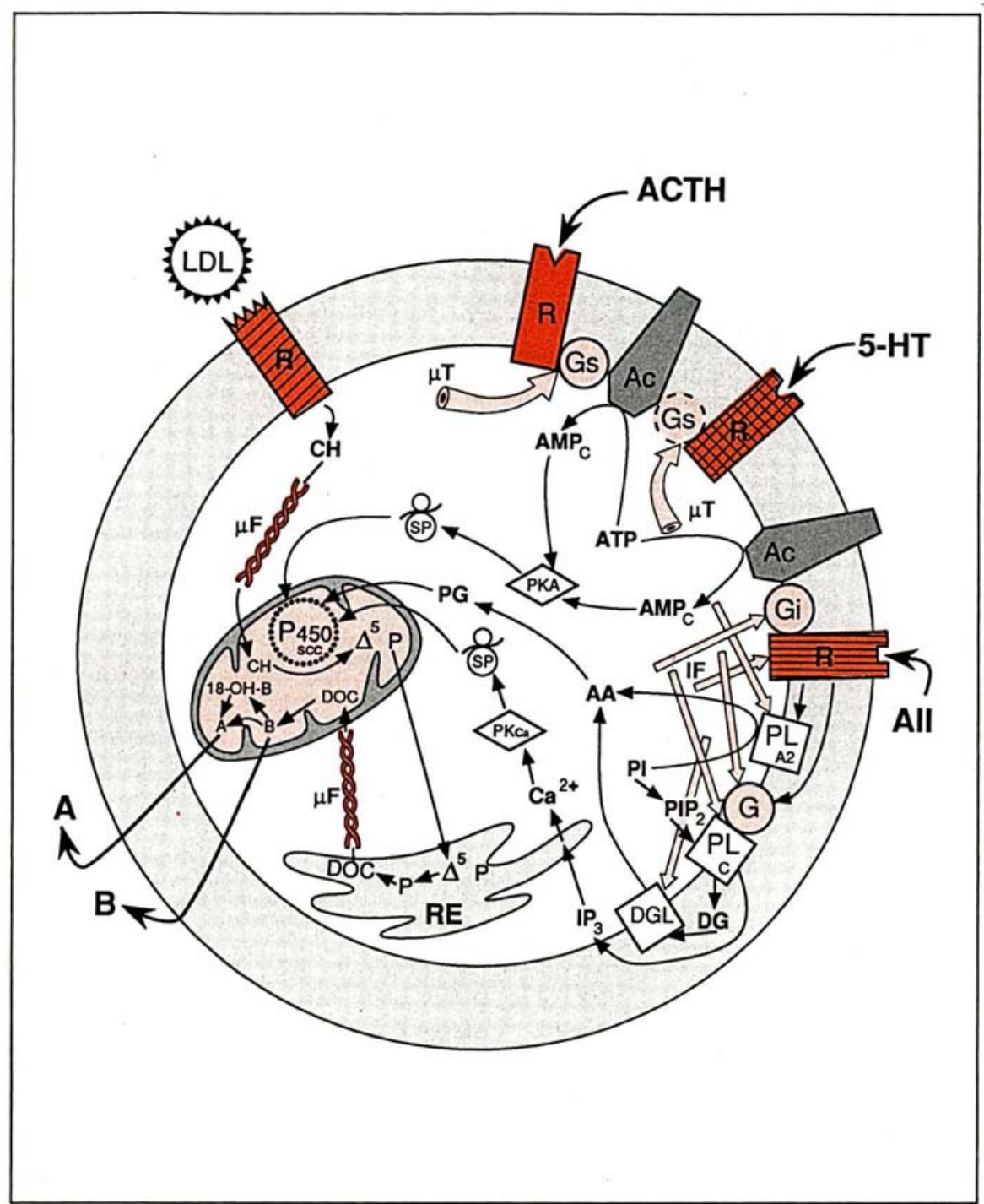

Figure 1. Représentation schématique du rôle du cytosquelette dans les cellules corticosurrénaliennes. Les microtubules sont impliqués dans la réponse des cellules à l'ACTH et d̀ la sérotonine. A l'échelle moléculaire, le site d'action des microtubules se situe probablement au niveau du couplage récepteur/ sous-unité régulatrice positive de l'adénylate cyclase. Les microfilaments contrôlent le transfert à la mitochondrie du cholestérol exogène et la translocation de la 11 désoxycorticostérone depuis le réticulum endoplasmique jusqu'à la mitochondrie. Les filaments intermédiaires paraissent impliqués dans le mécanisme d'action de l'angiotensine II, entre le couplage de l'hormone d̀ son récepteur et la production des seconds messagers. $A=$ aldostérone; $A A=$ acide arachidonique; $A \|$ II = angiotensine II; $A c=$ adénylate cyclase; $A C T H=$ hormone corticotrope hypophysaire; $A M P C=$ adénosine monophosphate cyclique $;$ ATP $=$ adénosine triphosphate; $B=$ corticostérone; $\mathrm{CH}=$ cholestérol; $D G=$ diacylglycérol; $D G L=$ diacylglycérol lipase $; D O C=11$-désoxycorticostérone; $G=$ protéine de couplage GTP dépendante; $\mathrm{Gi}=$ sous-unité régulatrice négative de l'adénylate cyclase; Gs = sous-unité régulatrice positive de l'adénylate cyclase; 5-HT = sérotonine ; IF = filaments intermédiaires ; IP3 = inositol triphosphate ; $L D L=$ low density lipoprotein (lipoprotéine de faible poids moléculaire); $18-\mathrm{OH}-\mathrm{B}=18$ hydroxycorticostérone; $P=$ progestérone; P450scc $=$ cytochrome P450scc; $\Delta^{5 P}=$ prégnénolone $; P G=$ prostaglandines $; P I=$ phosphatidylinositol; $P I P_{2}=$ phosphatidylinositol diphosphate; PKA = protéine kinase $A ; P K C a=$ protéine kinase calcium dépendante ; $P L A_{2}=$ phospholipase $A_{2} ; P L C=$ phospholipase $C$; $S P=$ synthèse protéique; $R=$ protéine réceptrice; $\mu F=$ microfilaments ; $\mu T=$ microtubules. des espèces, les cellules de la granulosa ne contiennent que des filaments intermédiaires de vimentine qui semblent en contact avec les récepteurs membranaires et les vésicules d'endocytose. Chez l'homme, ces cellules expriment à la fois des filaments de vimentine et de cytokératine dans un rapport qui dépend de leur état de stimulation [7]. Les filaments intermédiaires pourraient donc être impliqués dans la réponse des cellules stéroïdogènes aux facteurs de stimulation. Toutefois, jusqu'à présent, le rôle de ces fibres dans la sécrétion des hormones stéroïdes n'a été exploré en détail que dans la surrénale.

Dans les cellules corticosurrénaliennes, les filaments intermédiaires - ne sont composés que de cytokératine et organisés en un réseau dont l'importance varie selon les espèces. A l'inverse, les cellules tumorales issues du cortex surrénalien contiennent généralement très peu de filaments de kératine, mais expriment des fibres de vimentine. Malgré ces différences, l'IDPN, le seul agent dénaturant spécifique des filaments intermédiaires, n'a aucun effet sur la production spontanée de corticostéroïdes par les cellules surrénaliennes normales ou tumorales. Dans les cellules tumorales Yl, l'IDPN potentialise l'action de l'ACTH alors qu'il l'inhibe dans les cellules surrénalicnnes normales de rat et de poulet $[8,9]$. Des résultats récents montrent que les filaments intermédiaires sont impliqués dans le mécanisme d'action de l'angiotensine II, entre le couplage de l'hormone à son récepteur et la production de son second messager [10]. Il apparaît donc que le rôle des filaments intermédiaires dans la stéroïdogenèse varie considérablement selon les types cellulaires.

\section{Conclusion}

Dans les cellules stéroïdogènes, le cytosquelette apparaît ainsi comme une composante importante dans les mécanismes de transfert de l'information au niveau de la membrane cytoplasmique et de transport intracellulaire des substrats nécessaires au maintien de l'activité endocrinienne (figure 1). La spécificité d'espèce de la réponse de la plupart des types cellulaires aux agents de dépolymé- 
risation du cytosquelette rend toutefois difficile l'extrapolation à l'homme des résultats obtenus chez les animaux. En outre, l'étude clinique de l'activité de drogues telles que la cytochalasine B ou l'IDPN sur la production des stéroïdes est rendue pratiquement impossible par la toxicité de ces molécules. Le développement actuel de modèles d'études in vitro de cellules stéroïdogènes humaines normales permettra probablement de préciser le rôle des différents éléments du cytosquelette dans la biosynthèse des stéroïdes chez l'homme

M. Feuilloley : maître de conférences à l'université de Rouen, IUT d'Évreux.

P. Netchitaïlo : chargé de recherche au Cnrs.

C. Delarue: chargée de recherche au Cnrs.

F. Leboulenger : ingénieur d'université.

H. Vaudry: directeur de recherche à l'Inserm.

Groupe de recherche en endocrinologie moléculaire, Cnrs URA 650, unité affiliée à l'Inserm, université de Rouen, 76134 Mont-Saint-Aignan, France.

G. Pelletier : professeur au centre hospitalier de l'université Laval.

Centre de recherche en endocrinologie moléculaire, centre hospitalier de l'université Laval, Québec, G1V 4G2, Canada.

\section{RÉFÉRENCES}

1. Saltarelli D, De La Llossa-Hermier MP, Tertrin-Clary C, Hermier C. Effect of antimicrotubular agents in cAMP production and in steroidogenic response of isolated rat Leydig cells. Biol Cell 1984 ; 52 : 259-66.

2. Clark MA, Shay JW. The role of tubulin in the steroidogenic response of murine adrenal and rat Leydig cells. Endocrinology 1981 ; 109: 2261-3.

3. Feuilloley M, Netchitaïlo P, Lihrmann I, Vaudry $H$. Effect of vinblastine, a potent antimicrotubular agent on steroid secretion by perifused frog adrenal glands. J Steroid Biochem 1986 ; 25 : 143-7.

$\mathrm{m} / \mathrm{s} n^{\circ} 9$ vol. 5 , novembre 89
4. Hall PF, Nakamura M, Mrotek JJ. The actions of various cytochalasins on mouse adrenal tumor cells in relation to trophic stimulation of steroidogenesis. Biochim Biophys Acta 1981 ; 676 : 338-44.

5. Crivello JF, Jefcoate CR. Intracellular movement of cholesterol in rat adrenal cells. J Biol Chem 1980 ; 255 : 8144-51.

6. Feuilloley $M$, Netchitaïlo $P$, Delarue $C$, Lihrmann I, Vaudry H. Formation of $11 \beta$. hydroxysteroids requires the integrity of the microfilament network in adrenocortical cells. Biochem Biophys Res Commun 1987 ; $148: 1354-62$.

7. Ben-Ze'ev A, Amsterdam A. Regulation of cytoskeletal protein organization and expression in human granulosa cells in response to gonadotropin treatment. Endocrinology 1989: $124: 1033-41$

8. Lee HS, Mrotek JJ. The effect of intermediate filament inhibitors on steroidogenesis and cytoskeleton in $\mathrm{Yl}$ mouse adrenal tumor cells. Cell Biol Int Reports $1984 ; 8$ : 463-82. 9. Carsia RV, Schwarz LA, Weber H. Effect of 3,3'-iminodipropionitrile (IDPN) on corticosteroidogenesis of isolated adrenocortical cells. Proc Soc Exp Biol Med 1987 ; 184 : 4617.

10. Feuilloley M, Netchitaïlo P, Delarue C, Leboulenger $F$, Benyamina $M$, Vaudry $H$. Effect of the intermediate filament inhibitor IDPN on steroid secretion by frog adrenal glands. J Steroid Biochem 1988 ; 29: 439-42.

\section{Summary}

What are the roles of the cytoskeleton in the process of steroïd hormone secretion?

It is generally accepted that steroid hormones are not packaged in discrete organelles. However, several studies indicate that, in steroidogenic cells, cytoskeleton elements are involved in the process of hormonal secretion. The aim of the present review is to propose a general scheme concerning the role of the cytoskeleton in the biosynthesis and release of steroid hormones. It emerges that microtubules and intermediate filaments participate in the transduction of the messages at the level of the cytoplasmic membrane while microfilaments appear to be involved in steroid hormone biosynthesis by controlling transport of substrates, such as cholesterol and desoxycorticosterone, towards the mitochondria.

\section{TIRÉS A PART}

H. Vaudry. 